\title{
Influence of buoyancy effects on the mixing process and RTD in a side injection reactor equipped with Static Mixers
}

\author{
Jody Albertazzi, Federico Florit, Valentina Busini, ${ }^{*}$ and Renato Rota \\ Politecnico di Milano, Department of Chemistry, Materials and Chemical Engineering \\ "G. Natta", Piazza Leonardo da Vinci 32, 20133, Milano, Italy \\ E-mail: valentina.busini@polimi.it
}

\begin{abstract}
The mixing process and the residence time distribution $(R T D)$ of molecules inside reactors is a well known topic in chemical engineering: good radial mixing and poor axial mixing of chemical species are the essential conditions in order to achieve a plug flow behaviour in a tubular reactor, which is often highly desirable. While the influence on mixing and $R T D$ of viscosity and spatial velocities has been investigated in the literature, the influence of density differences between the streams to be mixed has been much less investigated, especially considering laminar regimes. Thus, the mixing and $R T D$ of two miscible liquids with different densities and viscosities in a side-injection tubular reactor equipped with Sulzer Static Mixers were studied by RANS-based CFD simulations. The results obtained show that, if adequate configurations are used, it is possible to well approximate radial mixing and a plug-flow behaviour, even when large differences in densities are involved. Moreover, graphics for a fast estimation of the maximum mixing length involved as well as for the corresponding $P e^{-1}$ value were obtained as a function of the Re number.
\end{abstract}




\section{Introduction}

The transition of batch or semi-batch processes to continuously conducted ones has been studied in recent years ${ }^{1,2}$ : indeed, even if discontinuous systems present great flexibility and are frequently employed in the fine chemical industry, for example for the production of polymers and pharmaceuticals ${ }^{3,4}$, a continuous process generally requires reduced volumes and can ensure higher productivity, enhanced heat transfer and a constant quality of the produced chemicals, since transient operations, such as charge and discharge of the reactor, are avoided ${ }^{2,5}$. Among continuous reactors, tubular reactors guarantee better heat exchange with respect to tank reactors, because of their higher heat transfer area-to-volume ratio, and higher productivity.

Therefore, a kinetic-free methodology useful to rigorously transform a semi-batch process into a continuous one has been recently $\operatorname{proposed}^{6-9}$ : it involves a series of side injection tubular reactors in which a plug-flow behaviour must be attained.

In order to achieve a plug flow behaviour inside a tubular reactor, complete radial mixing and no axial dispersion should be simultaneously achieved. These conditions are generally verified in an empty pipe for fully developed turbulent flow ( $R e>4000$, being $R e$ the Reynolds number $)^{10}$ and when fluids of equal density should be mixed (that is, when the buoyancy and viscous forces are negligible). This is feasible only for short residence times, since it usually requires high velocities to satisfy the full turbulence requirement. Moreover, many industrial processes, ranging from the petrochemical and food industry to the polymer and pharmaceuticals production, involve the blending of liquids of different densities ${ }^{11-13}$ in a laminar regime, since high reaction times are needed.

In these cases, the mixing process among the streams of reactant is based only on diffusion ${ }^{14,15}$ and often the resulting mixing times are too large, causing undesirable situations in terms of process planning, productivity levels and product quality control ${ }^{13}$; considering an empty tube, a plug flow behaviour can be obtained only if the tube is extremely long with respect to its diameter ${ }^{15,16}$, and possibly it follows a coiled geometry ${ }^{17,18}$. 
Alternatively, active mixers such as ultrasonic micromixers and pulse-flow mixers can be employed, but their efficiency is hindered by their high cost, difficult maintenance and high failure rate ${ }^{19}$. Therefore, static mixers (SMs), represent an alternative solution to dynamic mixers because of their simplicity and reduced cost. These devices induce a chaotic flow, thus favouring the mixing of chemical species, and require only the energy deriving from the flow momentum itself. Moreover, since they have no moving parts, their failure rate and maintenance costs are lower than active mixers and need reduced maintenance in comparison to the active mixers: for these reasons, SMs can be considered key instruments in the transition from discontinuous to continuous processes ${ }^{20}$.

Over the years, a wide range of SMs with different geometries has been designed and produced: considering laminar flows, the SMX Sulzer Static Mixer is reported to achieve the best performance in terms of radial mixing, as it is specifically designed to handle fluids having very different properties in terms of viscosities and flow rates ${ }^{21}$.

In a previous work, it has been shown that a side injection tubular reactor equipped with SMX SMs, in which equal densities fluids have to be mixed, is able to reach a plug flow behaviour (even considering Stokes flows with $R e<1$ ), in a few diameters from the lateral injection point depending on $R e^{22}$. Nevertheless, mixing fluids of different densities through a lateral injection in a tubular reactor equipped with SMs, is a much more complex problem, since also buoyancy forces can play a relevant role. In this case, not only Re but also Richardson number $(R i)$, which represents the ratio between buoyancy and inertial forces and the Grashof number $(G r)$, defined as the ratio between buoyancy forces and viscous forces, can have a major impact ${ }^{11,12,20}$.

While much information is available on pressure drops in static mixers ${ }^{20,21}$, detailed studies on mixing mechanisms are less common, mainly involving the influence of viscosity ${ }^{23}$ and buoyancy effects in the turbulent flow regime ${ }^{20}$. However, the mixing mechanisms and Residence Time Distribution $(R T D)$ of fluids having different densities in a side injection tubular reactor, operating in the laminar flow regime, is not fully investigated yet, even if it has been 
reported that the buoyancy forces can negatively impact on the mixing process even more than the viscosity forces ${ }^{11}$.

Therefore, the main aim of this work is to study, through Computational Fluid Dynamics (CFD) simulations, the mixing process and $R T D$ of a side injection tubular reactor equipped with SMX SMs in which two streams with different densities have to be mixed.

\section{Methods}

\section{Side injection tubular reactor geometry}

The simulated geometry consists of a side-injection tubular reactor equipped with a series of 27 SMX SMs, as reported in Figure 1: each static mixer involves an array of crossed bars, rotated with an angle of $45^{\circ}$ with respect to the pipe axis, and each element is rotated of $90^{\circ}$ with respect to the previous one.

The reactor involves a main pipe, having a diameter of $D_{a x}=5.08 \mathrm{~cm}$ and a length of $L_{a x}=1.3916 \mathrm{~m}$, and a lateral injection pipe, with a diameter of $D_{l a t}=1.27 \mathrm{~cm}$ and a length of $L_{l a t}=2.54 \mathrm{~cm}$.

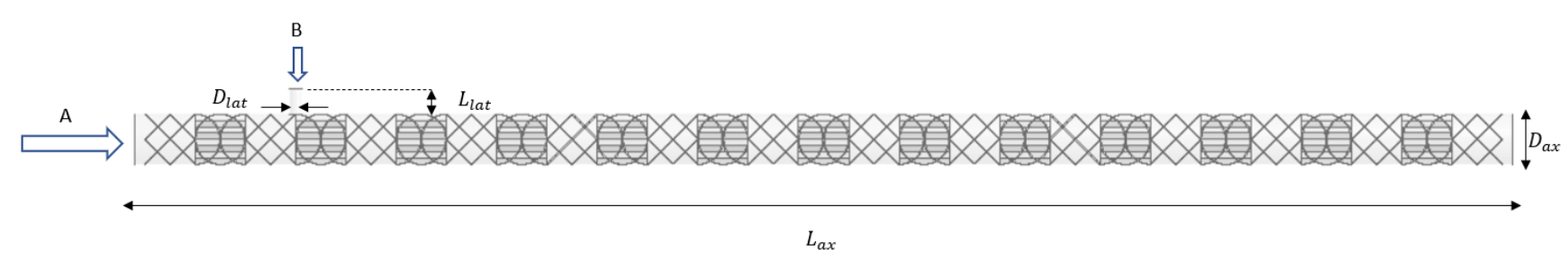

Figure 1: Side view of the reactor.

The two streams labelled A and B in Figure 1 can have very different characteristics, summarized in the values of Reynolds, Richardson, and Grashof numbers of the stream 
resulting from their mixing:

$$
\begin{gathered}
R e=\frac{\rho_{\text {mix }} \cdot v \cdot D_{a x}}{\mu_{\text {mix }}} \\
R i=\frac{g \cdot|\Delta \rho| \cdot D_{a x}}{\rho_{\text {mix }} \cdot v^{2}} \\
G r=\frac{g \cdot|\Delta \rho| \cdot \rho_{\text {mix }} \cdot D_{a x}^{3}}{\mu_{\text {mix }}^{2}}
\end{gathered}
$$

The physical quantities appearing in these equations are: the difference in density between the fluids $(\Delta \rho)$, the mixture density $\left(\rho_{m i x}\right)$, the mixture viscosity $\left(\mu_{m i x}\right)$, the spatial velocity $(v)$, the gravitational acceleration $(g)$ and, implicitly, the mass fractions of $\mathrm{A}\left(w_{A}\right)$ and $\mathrm{B}$ $\left(w_{B}\right)$ and they are computed as summarized in Table 1.

Table 1: Physical quantities appearing in $R e, R i, G r$.

\begin{tabular}{ccccccc}
\hline$\Delta \rho\left[\frac{\mathrm{kg}}{\mathrm{m}^{3}}\right]$ & $\rho_{\text {mix }}\left[\frac{\mathrm{kg}}{\mathrm{m}^{3}}\right]$ & $\mu_{m i x}[\mathrm{cP}]$ & $v\left[\frac{\mathrm{m}}{\mathrm{s}}\right]$ & $\omega_{A}$ & $\omega_{B}$ & $g\left[\frac{\mathrm{m}}{\mathrm{s}^{2}}\right]$ \\
\hline$\rho_{A}-\rho_{B}$ & $\left(\frac{\omega_{A}}{\rho_{A}}+\frac{\omega_{B}}{\rho_{B}}\right)^{-1}$ & $\omega_{A} \cdot \mu_{A}+\omega_{B} \cdot \mu_{B}$ & $\frac{4\left(m_{A}+m_{B}\right)}{\rho_{m i x} \cdot \pi \cdot D_{a x}^{2}}$ & $\frac{m_{A}}{\left(m_{A}+m_{B}\right)}$ & $\frac{m_{B}}{\left(m_{A}+m_{B}\right)}$ & 9.81 \\
\hline
\end{tabular}

\section{Mixing length estimation}

The mixing of the two streams were characterized by the coefficient of variation ${ }^{22}$, computed as reported in Equation 4:

$$
\operatorname{COV}(x)=\frac{\sqrt{\left(\overline{\omega_{B, x}^{2}}-\overline{\omega_{B, x}}\right)}}{\overline{\omega_{B, x}}}
$$

where $\overline{\omega_{B, x}^{2}}$ is the mass-flow average (on a cross section of the reactor) of the squared mass fraction of $B$ calculated at the axial position $x$ and $\overline{\omega_{B, x}}$ the mass-flow average (on the same section) of the mass fraction of $B$ calculated at the axial position $x$. Through the 
computation of the coefficient of variation at different axial locations it is possible to estimate the segregation of the two streams across the axial length: a high value of $C O V$ represents almost complete segregation of the two streams, while a value of $C O V$ equal to 0 represents complete mixing. Usually, for values equal or lower to 0.05 , complete mixing is assumed. Therefore, the mixing length between the two streams can be defined, in terms of the number of reactor diameters, as the distance between the injection point required to achieve $C O V=$ 0.05 .

\section{Residence time distribution estimation}

One method commonly used to study the $R T D$ of a reactor is the step injection experiment ${ }^{24}$, in which a tracer, with a step input, is injected in the stream flowing in the reactor. By measuring the tracer mass fraction in the stream leaving the reactor, the cumulative distribution function $(C D F)$ is obtained and through numerical differentiation of the $C D F$ the $R T D$ curve is computed ${ }^{15}$.

The same approach was used in this study: after running a steady-state simulation involving only the streams $A$ and $B$, the tracer $C$, having the same properties of the stream $B$, was injected with a step input and the transient simulation was started using a First Order Implicit Scheme.

The mass-weighted average of $C$ was monitored at the exit of the reactor and its $C D F$ was directly obtained. Therefore, the dimensionless $R T D$ curve was derived through numerical differentiation and normalization with the mean residence time $\bar{t}$ of the $C D F$ curve. Moreover, following an approach similar to the one adopted to obtain the $C O V$, the spatial evolution along the axial direction of the dimensionless $R T D$ was investigated.

To compare the performance of the simulated reactor with a PFR, both the Tanks-in-Series model $(T I S M)$ and the Axial-Dispersion-Model $(A D M)$ were used; the analytical solution of these models gives the number of equivalent CSTRs in series (the higher this number, the better the simulated reactor approximates the plug flow behaviour) and the dispersion 
number (the lower this number, the better the performance of the simulated reactor approximates the the PFR behaviour) ${ }^{15}$. In particular, the dispersion number represents the degree of dispersion along the axial coordinate and can be considered complementary to the COV (which represents the degree of dispersion along the radial coordinate): this number is defined as $\Theta=1 / P e$, where $P e$ is the Peclet number, which represents the ratio between the advective transport rate (given by the product between the considered length $L$ and the spatial velocoty $v$ ) and the diffusion transport rate (given by the mass diffusion coefficient $\mathscr{D}): P e=L v / \mathscr{D}$.

\section{CFD simulations}

In this work, Ansys Fluent 19.1 suite of programs was used to run RANS-based simulations by solving the continuity, momentum and species mass transport equations. Therefore, a turbulence closure model was also needed. The Shear-Stress Transport (SST) $k-\omega$ model was selected, since it can reproduce the transition from laminar to turbulent flow regimes ${ }^{25}$. The unstructured mesh used is composed by tetrahedral cells: because of the complex geometries of the static mixers, grid refinement close to the walls and inside the mixing elements was enforced: moreover, in order to have grid independent results, a mesh-independence test, which gives the minimum number of cells needed to conduct reliable simulations, needs to be performed.

Table 2: Boundary conditions for grid-independence test.

\begin{tabular}{cccccc}
\hline$\rho_{A}\left[\frac{\mathrm{kg}}{\mathrm{m}^{3}}\right]$ & $\rho_{B}\left[\frac{\mathrm{kg}}{\mathrm{m}^{3}}\right]$ & $\mu_{A}[\mathrm{cP}]$ & $\mu_{B}[\mathrm{cP}]$ & $m_{A}\left[\frac{\mathrm{g}}{\mathrm{s}}\right]$ & $m_{B}\left[\frac{\mathrm{g}}{\mathrm{s}}\right]$ \\
\hline 750 & 1000 & 10 & 1.0 & 90 & 9.0 \\
\hline
\end{tabular}

In a previous work, on the mixing of equal density fluids in SMX in the same reactor, a mesh-independence analysis was performed, finding that a mesh with cell dimensions of $5 \mathrm{~mm}$ and $1 \mathrm{~mm}$ near the walls was able to effectively reproduce the flow motion inside the static mixers $^{22}$ : with these cell dimensions, the geometry considered in this work can be represented 
with a grid with 10 million of cells. To prove that this mesh can provide reliable results, the mesh-independence test was repeated running a simulation (whose boundary conditions are reported in Table 2) and comparing the mixing lengths obtained with grids having 10 and 20 million of cells: the obtained results were 4.75 and 4.78 diameters, respectively. This indicates that from 10 million cells the results are independent from the mesh. As a further confirmation, since pressure drops are particularly susceptible to perturbations ${ }^{16}$, the pressure losses inside the reactor were compared to the ones obtained using an empirical correlation $^{26}$ reported in Equation 5:

$$
\Delta P=\frac{K_{L}}{R e} \cdot \rho \cdot v^{2} \cdot \frac{L}{D}
$$

where $K_{L}$ is a dimensionless factor and it varies in a range that goes from 820 to 1280 , depending on technical details such as the number of bars: for a standard Sulzer SMX with 8 bars, the $K_{L}$ factor can be assumed roughly equal to $1070^{21,26}$. The obtained results are reported in Figure 5, showing a good agreement between CFD and empirical correlation predictions. 


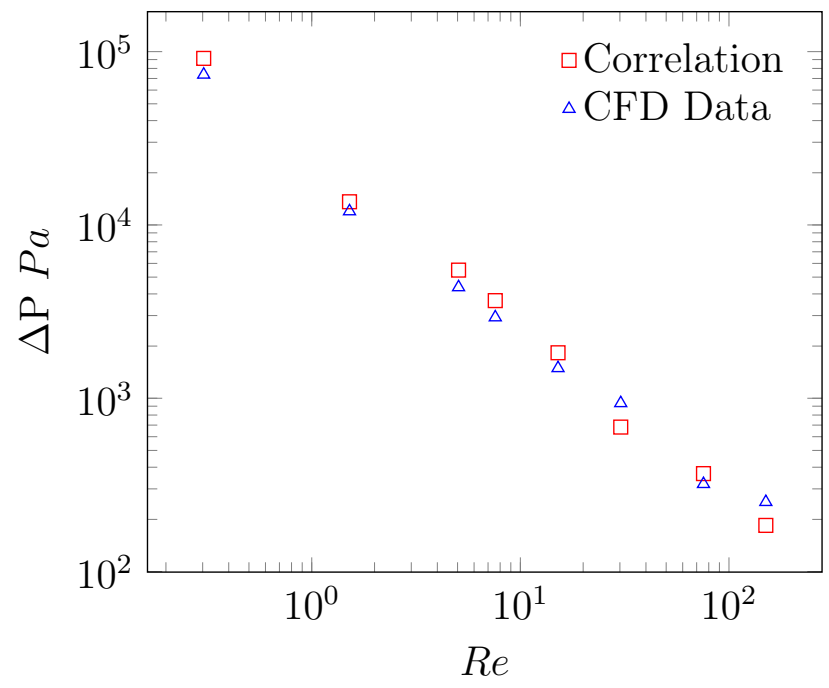

Figure 2: Comparison of pressure drop estimated through CFD simulations and the empirical correlation.

\section{Results and discussion}

\section{Mixing}

\section{Mixing in horizontal reactor}

In a previous work ${ }^{22}$, several simulations, aimed at assessing the mixing efficiency of a sideinjection tubular reactor equipped with the SMs, have been performed considering A and B streams with equal densities. It has been found that the mixing length depended only on the $R e$, ranging from 3 to 11 diameters.

To evaluate the influence of density variations on the mixing process a series of simulations were performed as summarized in Table 3.

Table 3: Boundary conditions and fluid properties.

\begin{tabular}{cccccc}
\hline$\rho_{A}\left[\frac{k g}{m^{3}}\right]$ & $\rho_{B}\left[\frac{k g}{m^{3}}\right]$ & $\mu_{A}[\mathrm{cP}]$ & $\mu_{B}[\mathrm{cP}]$ & $\dot{m}_{A}\left[\frac{g}{s}\right]$ & $\dot{m}_{B}\left[\frac{g}{s}\right]$ \\
\hline 750 & 1000 & 1.0 & 1.0 & 50 & 5.0 \\
750 & 1000 & 1.0 & 1.0 & 60 & 6.0 \\
750 & 1000 & 10 & 1.0 & 30 & 3.0
\end{tabular}




\begin{tabular}{|c|c|c|c|c|c|}
\hline 750 & 1000 & 100 & 1.0 & 30 & 3.0 \\
\hline 750 & 1000 & 2.0 & 1.0 & 30 & 3.0 \\
\hline 750 & 1000 & 6.0 & 1.0 & 46 & 4.6 \\
\hline 750 & 1000 & 8.0 & 1.0 & 45 & 4.5 \\
\hline 750 & 1000 & 20 & 1.0 & 48 & 4.8 \\
\hline 750 & 1000 & 50 & 1.0 & 20 & 2.0 \\
\hline 750 & 1000 & 300 & 1.0 & 20 & 2.0 \\
\hline 750 & 1000 & 4.0 & 1.0 & 50 & 5.0 \\
\hline 750 & 1000 & 2.5 & 1.0 & 40 & 4.0 \\
\hline 1000 & 1250 & 500 & 1.0 & 20 & 2.0 \\
\hline 1000 & 1250 & 500 & 1.0 & 35 & 3.5 \\
\hline 1000 & 1500 & 2.0 & 1.0 & 20 & 2.0 \\
\hline 1000 & 1500 & 3.5 & 1.0 & 80 & 8.0 \\
\hline 1000 & 1500 & 4.0 & 1.0 & 30 & 3.0 \\
\hline 1000 & 1500 & 0.25 & 1.0 & 20 & 2.0 \\
\hline 1000 & 750 & 100 & 1.0 & 80 & 8.0 \\
\hline 1000 & 750 & 1.0 & 1.0 & 75 & 7.5 \\
\hline 1000 & 750 & 2.0 & 1.0 & 60 & 6.0 \\
\hline 1000 & 750 & 1.0 & 1.0 & 35 & 3.5 \\
\hline 1000 & 750 & 1.0 & 1.0 & 45 & 4.5 \\
\hline 1000 & 750 & 500 & 1.0 & 35 & 3.5 \\
\hline
\end{tabular}

The obtained results are summarized in Figure 3 in terms of $L_{m i x} / D$ vs $R e$ number: we can see an apparently random behaviour of the mixing lengths, which are often much larger than the maximum mixing lengths obtained considering the equal density case (i.e., about 11 diameters). This behaviour can be attributed to the influence of the $R i$ number, which can hinder the mixing process, as discussed in the following. 


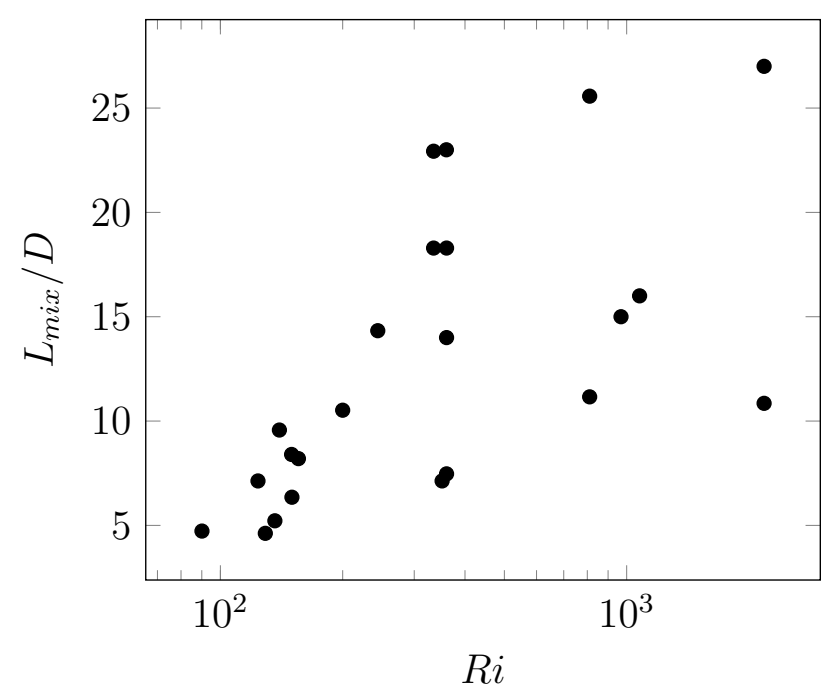

Figure 3: $L_{m i x} / D$ as a function of $R e$ for the horizontal reactor.

First configuration: $\rho_{A}<\rho_{B}$

In order to assess the influence of the $R i$ number on the mixing process, a series of simulations at constant Re (roughly equal to 250), were run while decreasing $R i$. Since the lateral injection is placed in the upper side of the axial pipe, the injected stream $B$ was assumed to have a higher density than the axial stream $A$, to possibly favour the penetration of the injected fluid through the axial fluid, thus enhancing the mixing.

In particular, $\rho_{A}=750 \mathrm{~kg} / \mathrm{m}^{3}$ and $\rho_{B}=1000 \mathrm{~kg} / \mathrm{m}^{3}$ were used. Other conditions for this set of simulations are reported in Table 4.

Table 4: Conditions for the first set of runs carried out at constant $R e$ and different $R i$.

\begin{tabular}{ccccc}
\hline$\mu_{A}[c P]$ & $\mu_{B}[c P]$ & $m_{A}\left[\frac{g}{s}\right]$ & $\dot{m}_{B}\left[\frac{g}{s}\right]$ & $R i$ \\
\hline 7.5 & 1.0 & 70 & 7.0 & 66 \\
8.5 & 1.0 & 60 & 6.0 & 90 \\
10 & 1.0 & 80 & 8.0 & 50 \\
15 & 1.0 & 130 & 13 & 20 \\
1.0 & 1.0 & 10 & 1.0 & 0 \\
\hline
\end{tabular}


The results sketched in Figure 4 show that when $R i$ is roughly lower than 50 the mixing length obtained becomes comparable to that determined in the equal density case.

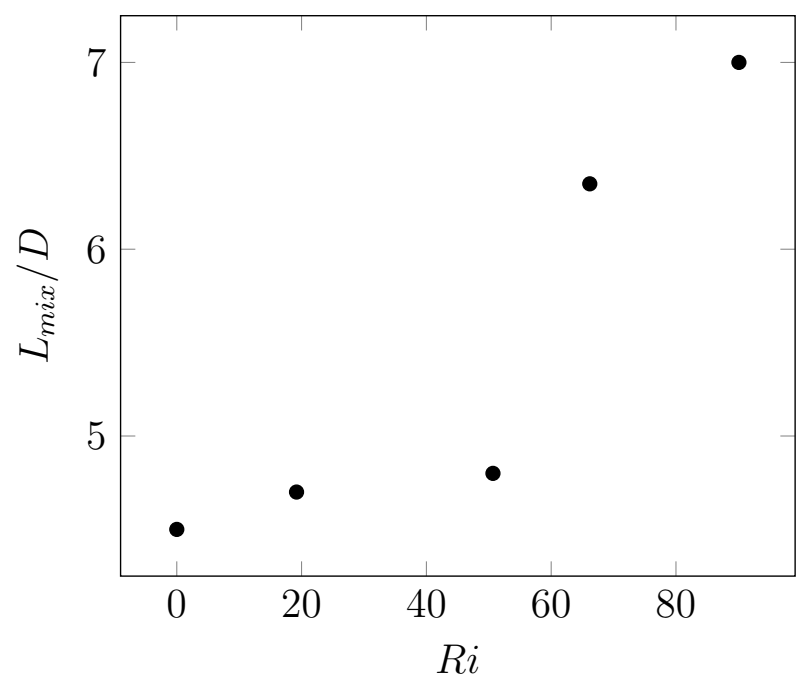

Figure 4: $L_{m i x} / D$ as a function of $R i$ at constant $R e$.

This was confirmed by a series of simulations run keeping $R i \leq 50$ while varying $R e$ as summarized in Table 5.

The obtained mixing lengths are compared in Figure 5 with the mixing lengths obtained for the equal density case (i.e., $R i=0)^{22}$. We can see that the results are similar with the exception of the region $3<R e<30$.

Therefore, as far as $R i<R i_{c r} \approx 50$ and $R e<3$ or $R e>30$, the mixing is not significantly influenced by the density of the two streams and the previous results obtained for the peculiar case of $\rho_{A}=\rho_{B}{ }^{22}$ can be used. On the contrary, when $R e \in[3,30]$ the mixing behaviour in terms of $L_{m i x} / D$ differs from the one found for the equal density case: however, in this range the mixing length predicted when $\rho_{A} \neq \rho_{B}$ is always smaller than the one when $\rho_{A}=\rho_{B}$. This means that the results previously obtained for $\rho_{A}=\rho_{B}$ can be safely used also in this range, since they represent a "worst case" in terms of mixing length.

Moreover, the value of $R i_{c r} \approx 50$ below which the SMX SMs are able to overcome the buoyancy forces, is much larger than the one found for other types of mixers such as the Kenics SMs ${ }^{27}$, thus confirming the high mixing efficiency of the SMX SMs. 
Table 5: Boundary conditions for the set of simulations with $R i<50$.

\begin{tabular}{|c|c|c|c|c|c|}
\hline$\rho_{A}\left[\frac{k g}{m^{3}}\right]$ & $\rho_{B}\left[\frac{k g}{m^{3}}\right]$ & $\mu_{A}[c P]$ & $\mu_{B}[c P]$ & $\dot{m}_{A}\left[\frac{g}{s}\right]$ & $\dot{m}_{B}\left[\frac{g}{s}\right.$ \\
\hline 750 & 1000 & 22 & 1.0 & 90 & 9.0 \\
\hline 750 & 1000 & 90 & 1.0 & 90 & 9.0 \\
\hline 750 & 1000 & 1.0 & 1.0 & 90 & 9.0 \\
\hline 750 & 1000 & 100 & 1.0 & 90 & 9.0 \\
\hline 750 & 1000 & 200 & 1.0 & 90 & 9.0 \\
\hline 750 & 1000 & 300 & 1.0 & 90 & 9.0 \\
\hline 750 & 1000 & 400 & 1.0 & 90 & 9.0 \\
\hline 750 & 1000 & 500 & 1.0 & 90 & 9.0 \\
\hline 750 & 1000 & 800 & 1.0 & 200 & 20 \\
\hline 750 & 1000 & 1000 & 1.0 & 90 & 9.0 \\
\hline 750 & 1000 & $1.0 \cdot 10^{4}$ & 1.0 & 90 & 9.0 \\
\hline 750 & 1000 & 10 & 1.0 & 90 & 9.0 \\
\hline 750 & 1000 & 15 & 1.0 & 90 & 9.0 \\
\hline 750 & 1000 & 20 & 1.0 & 90 & 9.0 \\
\hline 750 & 1000 & 30.5 & 1.0 & 90 & 9.0 \\
\hline 750 & 1000 & 50 & 1.0 & 90 & 9.0 \\
\hline 750 & 1000 & 9.0 & 1.0 & 90 & 9.0 \\
\hline 750 & 1000 & 7.5 & 1.0 & 90 & 9.0 \\
\hline 750 & 1000 & 5.0 & 1.0 & 90 & 9.0 \\
\hline 750 & 1000 & 4.0 & 1.0 & 150 & 150 \\
\hline 750 & 1000 & 1500 & 1.0 & 90 & 9.0 \\
\hline 1000 & 1500 & 90 & 1.0 & 140 & 14 \\
\hline 1000 & 1500 & $5.0 \cdot 10^{-1}$ & 1.0 & 200 & 20 \\
\hline 1000 & 1500 & 5.0 & 1.0 & 150 & 150 \\
\hline 1000 & 1500 & 1.5 & 1.0 & 130 & 13 \\
\hline
\end{tabular}




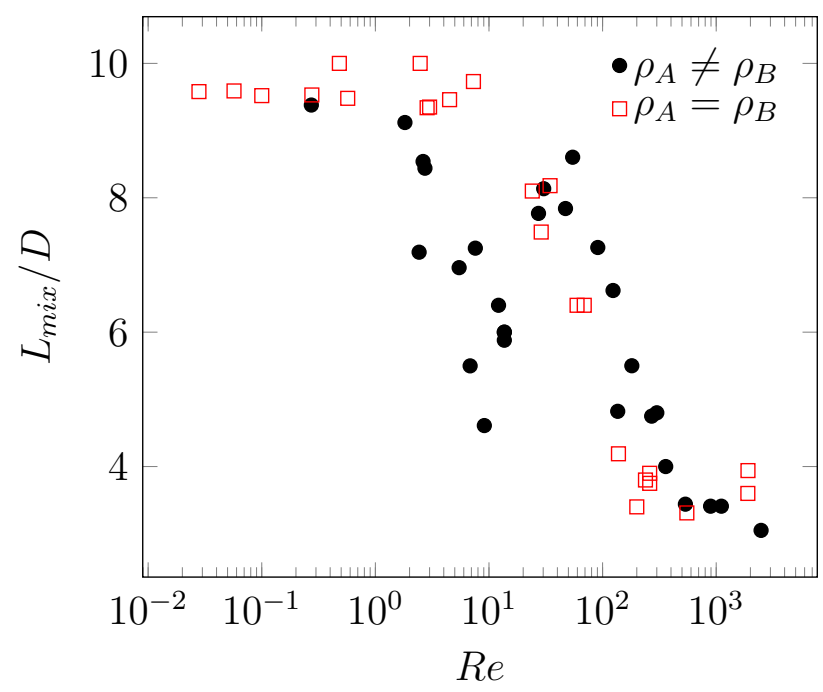

Figure 5: $L_{m i x} / D$ as a function of $\operatorname{Re}$ for $\rho_{A} \neq \rho_{B}$ and $\rho_{A}=\rho_{B}$. Data for $\rho_{A}=\rho_{B}$ from $^{22}$.

The points obtained when $\rho_{A} \neq \rho_{B}$ and $3<R e<30$ tend to form a minimum in the mixing length as a function of $R e$, which can be explained considering the influence of $G r$. Three different scenarios can be obtained:

- When $G r>1.2 \cdot 10^{5}$ the viscosity forces of the $A$ stream are not sufficient to avoid the stratification of the $B$ stream on the bottom of the reactor (Figure 6a), but since we are considering $R i \leq 50$ the SMX SMs are able to effectively overcome the buoyancy forces, thus obtaining mixing lengths which are comparable to the equal density case ${ }^{22}$.

- When $3.0 \cdot 10^{2}<G r<1.2 \cdot 10^{5}$ the most favourable scenario is obtained, since the $B$ stream penetrates the axial flow without stratifying on the bottom of the reactor, as reported in Figure 6b: in this case, the obtained mixing lengths are lower than the ones obtained in the equal density case ${ }^{22}$.

- When $G r<3 \cdot 10^{2}$, the viscosity forces of the $A$ stream are large enough to avoid the penetration of the $B$ stream, as reported in Figure 6c: in this case, since no stratification occurs, the buoyancy forces do not play a significant role and thus the obtained mixing length is comparable to the equal density case ${ }^{22}$. 
(6a)

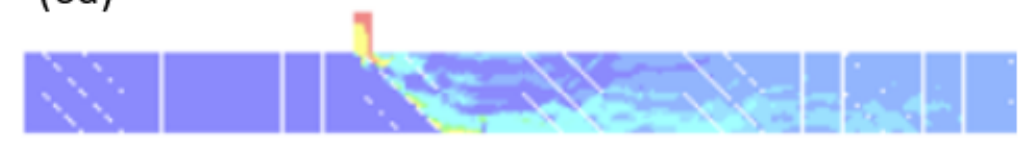

(6b)

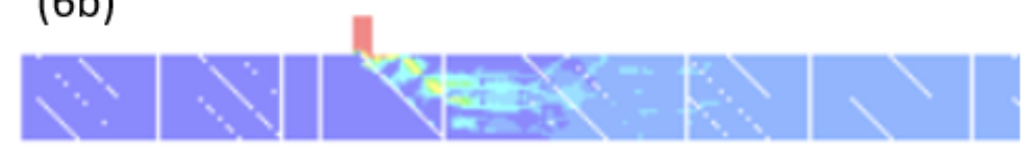

(6c)

Figure 6: Effect of $G r$ on mixing length. (6a): $G r>1.2 \cdot 10^{5} ;(6 \mathrm{~b}): G r \approx 3 \cdot 10^{3} ;(6 \mathrm{c})$ : Gr $<3 \cdot 10^{2}$.

A second set of simulations was carried out by varying the mass flow rate ratio of the two streams and the number of lateral inlets (as shown in Figure 7) as summarized in Table 6.

The obtained mixing lengths are reported in Figure 8, in which the mixing lengths obtained for one lateral inlet and $\dot{m}_{A} / m_{B}=10$ (previously shown in Figure 5) are also reported for comparison. We can see that outside the region of $R e \in[3,30]$ the mixing process is enhanced by the reduction of mass flow ratio between the two streams and by the increase of the number of lateral injections. This agrees with the results found for the equal density $\operatorname{case}^{22}$. 


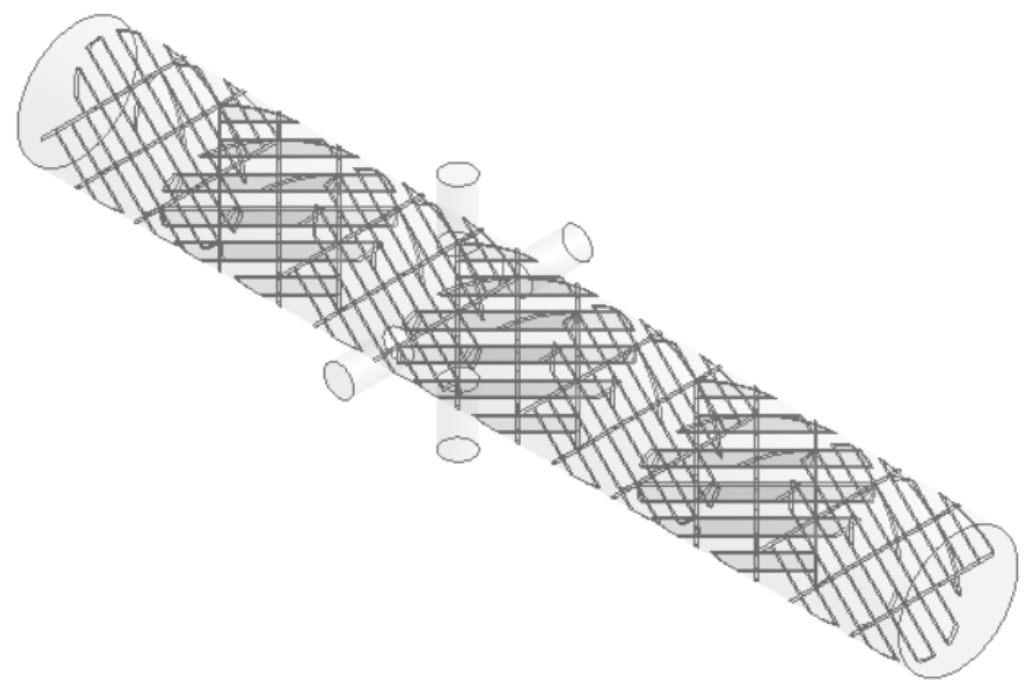

Figure 7: Detail of reactor geometry with four lateral inlets.

Table 6: Set-up of the tests carried out with $m_{A} / m_{B}=1$ or with four lateral inlets.

\begin{tabular}{ccccccc}
\hline$\rho_{A}\left[\frac{k g}{m^{3}}\right]$ & $\rho_{B}\left[\frac{k g}{m^{3}}\right]$ & $\mu_{A}[\mathrm{cP}]$ & $\mu_{B}[\mathrm{cP}]$ & $\dot{m}_{A}\left[\frac{g}{s}\right]$ & $\dot{m}_{B}\left[\frac{g}{s}\right]$ & Lateral Inlets \\
\hline 1000 & 1500 & $1.0 \cdot 10^{4}$ & 1.0 & 20 & 20 & 1 \\
1000 & 1500 & 1300 & 1.0 & 20 & 20 & 1 \\
1000 & 1500 & 200 & 1.0 & 20 & 20 & 1 \\
1000 & 1500 & 20 & 1.0 & 20 & 20 & 1 \\
750 & 1000 & 5.0 & 1.0 & 100 & 10 & 4 \\
750 & 1000 & 50 & 1.0 & 100 & 10 & 4 \\
750 & 1000 & 500 & 1.0 & 100 & 10 & 4 \\
750 & 1000 & 5000 & 1.0 & 100 & 10 & 4 \\
\hline
\end{tabular}




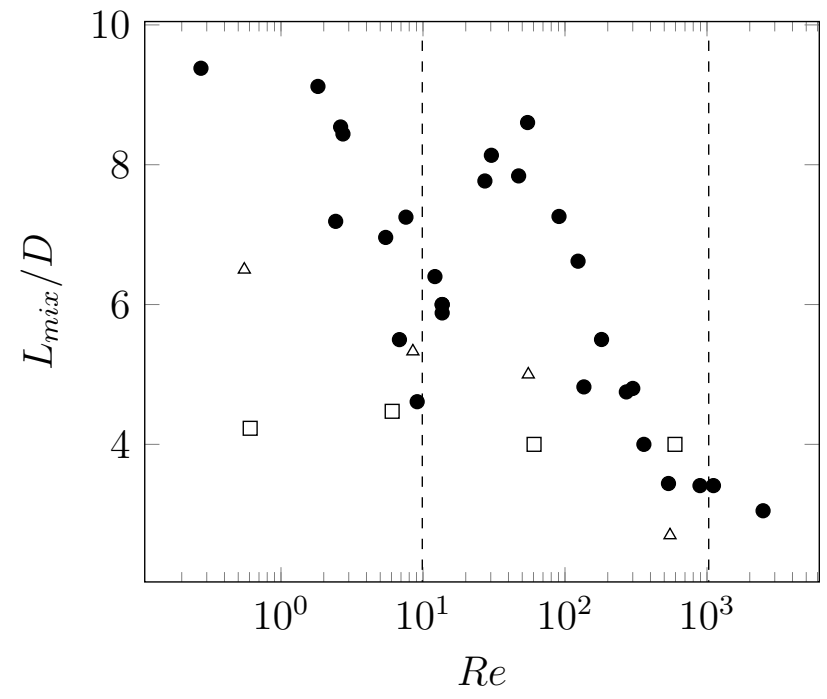

Figure 8: $(\bullet): \dot{m}_{A} / m_{B}=10$, one inlet; $(\triangle): \dot{m}_{A} / m_{B}=1$, one inlet; $(\square): \dot{m}_{A} / m_{B}=10$, four inlets.

\section{Second configuration: $\rho_{A}>\rho_{B}$}

Up to now the top-injected fluid was always assumed to be heavier than the axial one, exploiting gravity for the penetration of the lateral stream (and thus enhancing mixing). Considering the symmetry of the reactor, the same results obtained when $\rho_{A}<\rho_{B}$ are expected when $\rho_{A}>\rho_{B}$ provided that the lateral injection is positioned on the bottom of the axial tube, as reported in Figure 9.

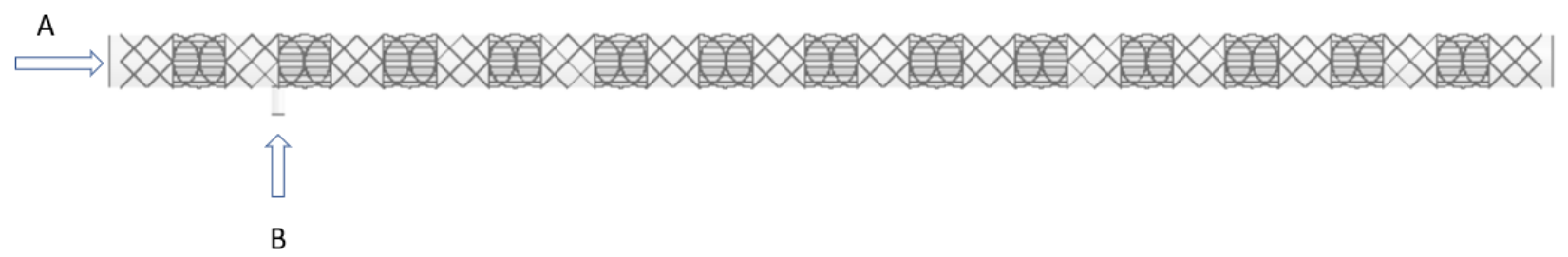

Figure 9: Reactor configuration when $\rho_{A}>\rho_{B}$.

A series of simulations, considering an injected stream $B$ such that $\rho_{B}>\rho_{A}$ and $0 \leq$ $R i \leq 50$ were performed. The densities were set equal to $\rho_{A}=1000 \mathrm{~kg} / \mathrm{m}^{3}$ and $\rho_{B}=750$ 
$\mathrm{kg} / \mathrm{m}^{3}$, the mass flow rates to $\dot{m}_{A}=90 \mathrm{~g} / \mathrm{s}$ and $\dot{m}_{B}=9.0 \mathrm{~g} / \mathrm{s}$, while the $A$ viscosity was equal to the values $\mu_{A}=\left[500 \cdot 10^{-2}, 1.0,100,1000,1.0 \cdot 10^{4}\right] c P$.

The obtained mixing lengths are compared in Figure 10 to the results obtained for $\rho_{A}<\rho_{B}$ : we can see that the mixing lengths are almost the same therefore confirming that the results obtained until now can be easily extended to the case $\rho_{A}>\rho_{B}$ if the lateral injection is located on the bottom instead of on the top.

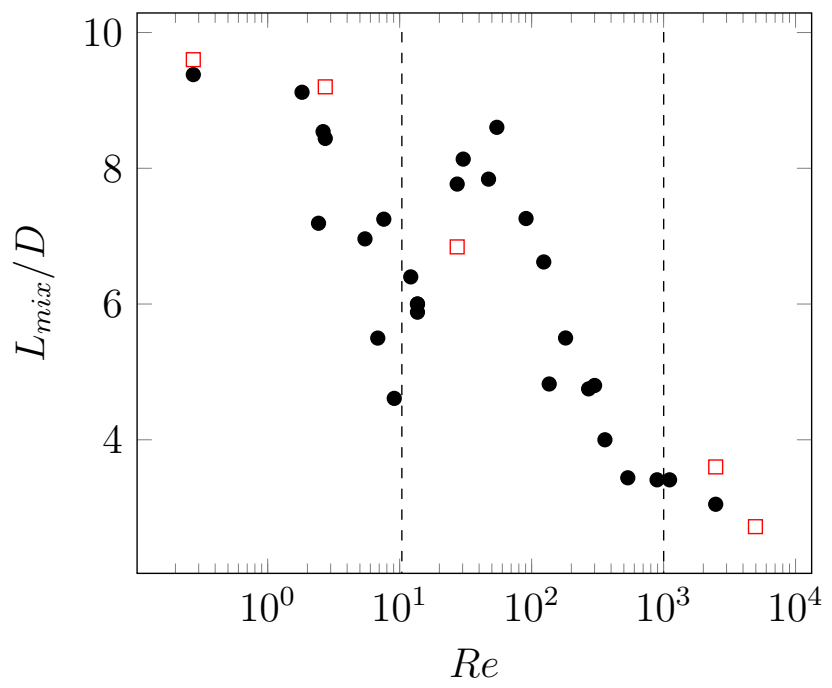

Figure 10: $L_{m i x} / D$ for $\rho_{A}>\rho_{B}$ and $\rho_{A}<\rho_{B}$.

\section{Mixing in vertical reactors}

As previously discussed, a horizontal reactor equipped with SMX Sulzer SMs can mix efficiently also two streams with different density only when $R i \leq 50$; otherwise, the mixing lengths can become impractically high and the side-injection tubular reactor does not reproduce any more the PFR behaviour.

Therefore, a series of simulations with $R i>50$ were run while changing the reactor axis direction, that is, considering a vertical reactor instead of a horizontal one in order to avoid the stratification of the injected fluid on the bottom or on the top of the reactor. The side injection position (as for the horizontal reactor) was properly located so as to favour the penetration of the injected fluid into the main flow and to avoid backflow. Therefore, when 
$\rho_{A}<\rho_{B}$ the side injection pipe was located on the top of the vertical reactor to favour the descent of the heavier fluid, while if $\rho_{A}>\rho_{B}$ the reactor will be "bottom to top" to enhance the rise of the lighter fluid, as sketched in Figure 11. Boundary conditions for this set of simulations are reported in Table 7.

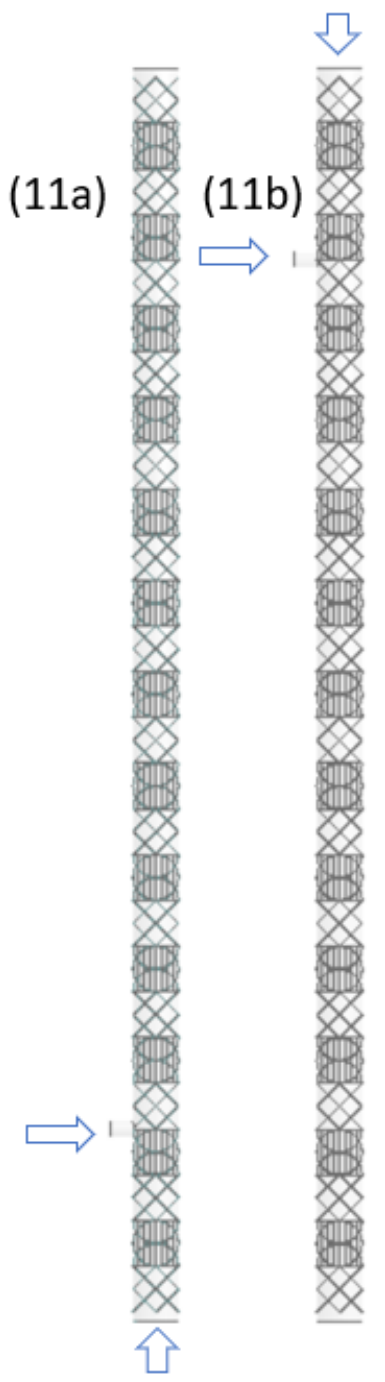

Figure 11: Reactor configuration for $\rho_{A}>\rho_{B}(11 \mathrm{a})$ and $\rho_{A}<\rho_{B}(11 \mathrm{~b})$

The mixing lengths obtained are compared to the results obtained in a horizontal reactor with equal density streams ${ }^{22}$ in Figure 12. We can see that, moving from horizontal to vertical, the behaviour predicted when mixing streams with different densities is much more similar to that of streams with equal density also for $R i$ values larger than the critical value of 50. This is coherent with a definition of $R i$ accounting for the main flow direction with 
Table 7: Set-up of the tests carried out with $R i>50$ in a vertical reactor.

\begin{tabular}{|c|c|c|c|c|c|}
\hline$\rho_{A}\left[\frac{k g}{m^{3}}\right]$ & $\rho_{B}\left[\frac{k g}{m^{3}}\right]$ & $\mu_{A}[\mathrm{cP}]$ & $\mu_{B}[\mathrm{cP}]$ & $\dot{m}_{A}\left[\frac{g}{s}\right]$ & $\dot{m}_{B}\left[\frac{g}{s}\right]$ \\
\hline 750 & 1000 & 400 & 1.0 & 100 & 10 \\
\hline 750 & 1000 & 50 & 1.0 & 20 & 2.0 \\
\hline 750 & 1500 & 10 & 1.0 & 20 & 2.0 \\
\hline 750 & 1000 & 10 & 1.0 & 3.0 & $3.0 \cdot 10^{-1}$ \\
\hline 750 & 1000 & 100 & 1.0 & 5.0 & $5.0 \cdot 10^{-1}$ \\
\hline 750 & 1000 & 100 & 1.0 & 10 & 1.0 \\
\hline 750 & 1000 & 500 & 1.0 & 50 & 5.0 \\
\hline 750 & 1000 & 500 & 1.0 & 500 & 50 \\
\hline 750 & 1000 & 500 & 1.0 & 5000 & 500 \\
\hline 750 & 1000 & 100 & 1.0 & 300 & 30 \\
\hline 1000 & 1500 & 10 & 1.0 & 10 & 1.0 \\
\hline 1000 & 1500 & 10 & 1.0 & 4.0 & $4.0 \cdot 10^{-1}$ \\
\hline 1000 & 1500 & 1000 & 1.0 & 10 & 1.0 \\
\hline 1000 & 1500 & 100 & 1.0 & 10 & 1.0 \\
\hline 1000 & 1500 & 1.0 & 1.0 & 10 & 1.0 \\
\hline 1000 & 1500 & 3000 & 1.0 & 15 & 1.5 \\
\hline 1000 & 1500 & 500 & 1.0 & $1.2 \cdot 10^{4}$ & 1200 \\
\hline 1000 & 1500 & 550 & 1.0 & 15 & 1.5 \\
\hline 1000 & 750 & 10 & 1.0 & 10 & 1.0 \\
\hline 1000 & 750 & 10 & 1.0 & 3.0 & $3.0 \cdot 10^{-1}$ \\
\hline 1000 & 750 & 1000 & 1.0 & 7.0 & $7.0 \cdot 10^{-1}$ \\
\hline 1000 & 750 & 1.0 & 1.0 & 7.0 & $7.0 \cdot 10^{-1}$ \\
\hline 1500 & 1000 & 1.0 & 1.0 & 20 & 2.0 \\
\hline 1500 & 1000 & 1.0 & 1.0 & 30 & 3.0 \\
\hline 1500 & 1000 & $5.0 \cdot 10^{-1}$ & 1.0 & 20 & 2.0 \\
\hline
\end{tabular}


respect to the vertical direction ${ }^{28}$, as reported in Equation 6:

$$
R i=\frac{g \cdot|\Delta \rho| \cdot D_{a x}}{\rho_{\text {mix }} \cdot v^{2}} \cdot \cos \alpha
$$

where $\alpha$ is the angle measuring the inclination of the reactor. Therefore, when the reactor is vertical, $\cos \left(90^{\circ}\right)=0$ and $R i=0$, leading to a behaviour similar to that of equal density streams. This is consistent with the fact that placing the reactor vertically, no work against gravity must be done to mix the chemical species since no stratification occurs. In particular, also in this case more scattered results were found in the range $R e \in[3,30]$. This can be once again due to the influence of $G r$, which measures the degree of penetration of the injected component into the axial flow: indeed, the larger the $G r$, the deeper the penetration of the injected stream into the main stream and thus the smaller the mixing length obtained. However, as for the previously discussed horizontal configuration when $R i<50$, the mixing lengths are always lower (or quite similar to) the results obtained for equal density streams. This means that also for vertical reactors the results previously obtained for $\rho_{A}=\rho_{B}$ can be safely used to foresee the "worst case" in terms of maximum mixing length.

\section{Residence time distribution analysis}

The previous analysis allows to estimate the mixing length expected in different operating conditions. By comparing such a mixing length with the reactor length, it is possible to decide whether SMX SMs can provide a reasonable approximation of a perfect mixing of the reactants at the entrance of the reactor. However, this is a necessary but not sufficient condition for considering the reactor behaviour to approximate the PFR one.

To assess if a reactor equipped with Sulzer SMX Static Mixers can approximate a PFR, a residence time distribution analysis must be also performed. To this aim, a series of simulations (considering both $R i \leq 50$ with the reactor oriented horizontally, and $R i \gg 50$ with the reactor positioned vertically), were performed as summarised in Table 8. 


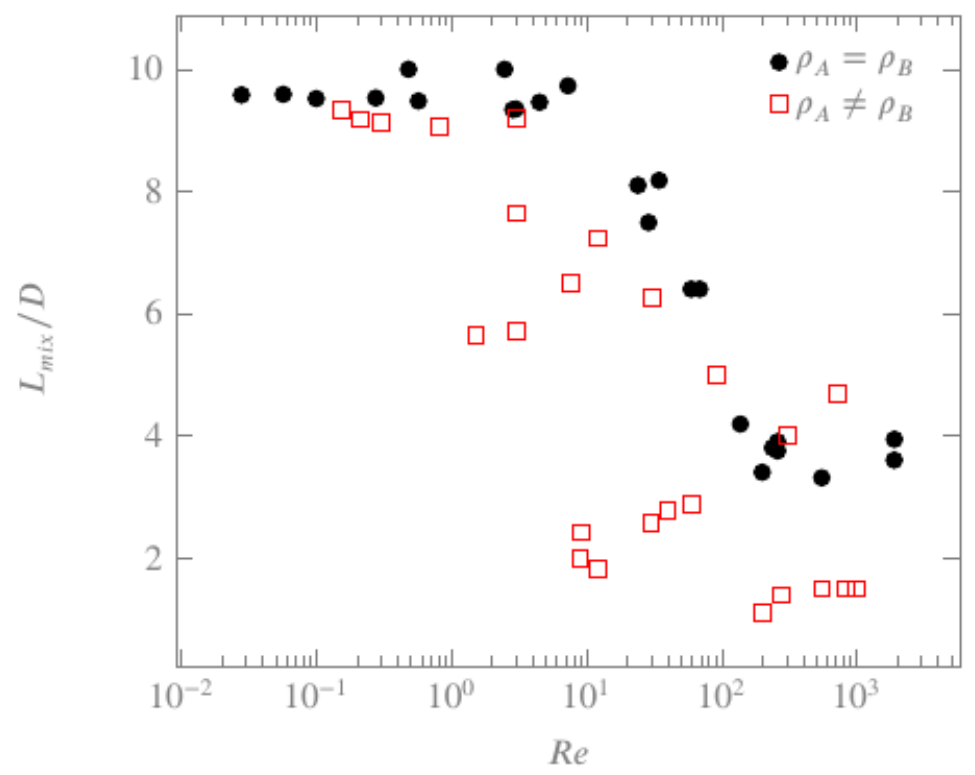

Figure 12: Mixing lengths obtained for $R i>50$ considering a reactor oriented vertically. Data for $\rho_{A}=\rho_{B}$ from $^{22}$.

As previously mentioned, once the $R T D$ curve was computed by differentiation of the CDF curve, the reactor performance was compared to that of an ideal PFR using both the TISM and the ADM. These models allow to estimate the number of CSTRs in series and the inverse of the Peclet number, both parameters being useful to estimate how the side-injection tubular reactor approximates a plug flow behaviour.

Table 8: Set-up and results of the tests carried out for the RTD analysis.

\begin{tabular}{ccccccccc}
\hline$\mu_{A}[c P]$ & $\mu_{B}[c P]$ & $\dot{m}_{A}\left[\frac{g}{s}\right]$ & $\dot{m}_{B}\left[\frac{g}{s}\right]$ & $P e^{-1}$ & $N_{C S T R s}$ & $V_{\text {dead }}[\%]$ & $R i$ & $R e$ \\
\hline 1.0 & 1.0 & 90 & 9.0 & $1.2 \cdot 10^{-2}$ & 33 & 4 & 40 & 2.7 \\
100 & 1.0 & 90 & 9.0 & $7.7 \cdot 10^{-2}$ & 34 & 4 & 40 & 27 \\
10 & 1.0 & 90 & 9.0 & $5.7 \cdot 10^{-3}$ & 60 & 3 & 40 & 270 \\
500 & 1.0 & 10 & 1.0 & $1.7 \cdot 10^{-2}$ & 33 & 5 & $6.3 \cdot 10^{4}$ & 6 \\
50 & 10 & 100 & 10 & $1.6 \cdot 10^{-2}$ & 35 & 5 & 638 & 60 \\
5.0 & 10 & 100 & 10 & $6.9 \cdot 10^{-3}$ & 78 & 4 & 638 & 600 \\
\hline
\end{tabular}

The results obtained, also reported in table 8 show that in all the conditions investigated the PFR behaviour is well reproduced by the side-injection reactor equipped with SMX 
SMs. This is also confirmed by the fraction of dead volumes, which represents the fraction of stagnant zones inside the reactor, computed as:

$$
V_{\text {dead }}[\%]=\frac{(\tau-\bar{t}) \cdot Q}{V_{\text {reactor }}}
$$

where $\tau$ is the ratio of the reactor volume to the total volumetric flowrate, $Q$ the volumetric flowrate and $V_{\text {reactor }}$ the reactor volume.

A plot correlating the $C O V$ (which is a measure of the radial dispersion) and the $P e^{-1}$ (which is a measure of the axial dispersion), as previously proposed ${ }^{22}$ is reported in Figure 13. We can see that the points are aligned in a monotonically increasing curve. Moreover, the points almost align with the points previously presented in literature for the equal density case $(R i=0)^{22}$, which are also reported in Figure 8 for the sake of comparison.

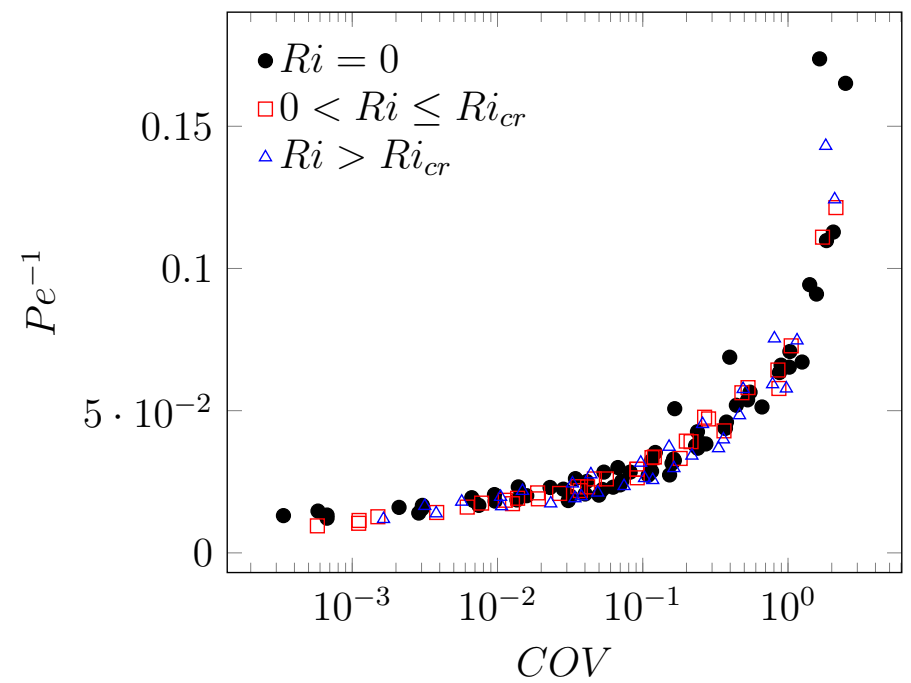

Figure 13: $P e^{-1}$ as a function of the coefficient of variation. $R i=0$ data from $^{22}$.

This means that once SMX SMs are able to guarantee a given value of the mixing length, thay are also able to guarantee a corresponding value of the axial dispersion, which can be easily estimated (as an order of magnitude) from Figure 13 in terms of $P e^{-1}$ values. 


\section{Conclusions}

In this study, a side-injection tubular reactor equipped with SMX SMs was modeled using a CFD code for investigating the mixing of streams with different densities, to assess the conditions allowing to approximate a plug flow behaviour.

It was found that when $R i<R i_{c r} \approx 50$ the reactor orientation (that is, horizontal or vertical) does not play a relevant role. However, when $R i>R i_{c r}$ the horizontal orientation can lead to very large mixing lengths. Moreover, considering only the optimal configuration (that is, horizontal with top injection when $\rho_{A}<\rho_{B}$ and with bottom injection when $\rho_{A}>\rho_{B}$; and vertical with falling flow when $\rho_{A}<\rho_{B}$ and with rising flow when $\rho_{B}<\rho_{A}$ ) the maximum mixing length can be easily estimated as a function of $R e$ using the diagrams previously determined for the $\rho_{A}=\rho_{B}$ case. From such a plot, it follows that the maximum mixing length is equal to about 11 diameters when $R e<10$ and to about 4 diameters when Re > 1000; a linear correlation can provide an estimation of the maximum mixing length when $10<R e<1000$. When these values of mixing length are higher than the acceptable threshold (which obviously depends on the reactor length), they can be further reduced by increasing the number of lateral injection points.

Finally, using the graph provided by Figure 13, from the mixing length value it is possible

to estimate the corresponding value of $P e^{-1}$, therefore allowing to understand if the sideinjection reactor can safely approximate a PFR or not, avoiding a long transient simulation of a tracer experiment.

\section{References}

(1) Kimura, H.; Tomatsu, K.; Saiki, H.; Arimitsu, K.; Ono, M.; Kawashima, H.; Iwata, R.; Nakanishi, I.; Ozeki, E.; Kuge, Y.; Saji, H. Continuous-Flow Synthesis of NSuccinimidyl 4-[18F] fluorobenzoate Using a Single Microfluidic Chip. Plos One 2016,

(2) Benaglia, M.; Puglisi, A.; Porta, R.; Coccia, F. Organocatalysis chemistry in flow. 
Current Organocatalysis 2015, 2, 79-101.

(3) Ilare, J.; Sponchioni, M.; Storti, G.; Moscatelli, D. From batch to continuous freeradical solution polymerization of acrylic acid using a stirred tank reactor. Reaction Chemistry \& Engineering 2020, 5, 2081-2090.

(4) Durand, A.; Engell, S. Batch to Conti Transfer of Polymer Production Processes. Macromolecular Reaction Engineering 2016, 10, 308-310.

(5) Asua, J., M. Challenges and Opportunities in Continuous Production of Emulsion Polymers: a Review. Macromolecular Reaction Engineering 2015, 10, 311-323.

(6) Florit, F.; Busini, V.; Storti, G.; Rota, R. From semi-batch to continuous tubular reactors: A kinetics-free approach. Chemical Engineering Journal 2018, 1007-1017.

(7) Florit, F.; Busini, V.; Storti, G.; Rota, R. Kinetics-free transformation from nonisothermal discontinuous to continuous tubular reactors. Chemical Engineering Journal 2019, 373, 792-802.

(8) Florit, F.; Busini, V.; Rota, R. Kinetics-free process intensification: From semi-batch to series of continuous chemical reactors. Chemical Engineering \& Processing 2020, $154,108014$.

(9) Maestri, F.; Copelli, S.; Barozzi, M.; Rota, R. Kinetic-free discontinuous to continuous transformation of fine chemical reactions: A general experimental procedure. Chemical Engineering Journal 2020, 395, 125061.

(10) Diyoke, C.; Ngwaka, U. CFD Analysis of a Fully Developed Turbulent Flow in a Pipe with a Constriction and an Obstacle. International Journal of Engineering Research \& Technology 2015, 4, 2278-0181.

(11) Bouwmans, I.; Bakker, A.; Van Der Akker, H. Blending Liquids of Differing Viscosities 
and Densities in Stirred Vessels. Chemical Engineering Research and Design 1997, 75, $777-783$.

(12) Derksen, J. Blending of miscible liquids with different densities starting from a stratified state. Computers \& Fluids 2011, 50, 35-45.

(13) Gogate, P. R.; Pandit, A. B. Mixing of Miscible Liquids with Density Differences: Effect of Volume and Density of the Tracer Fluid. The Canadian Journal of Chemical Engineering 1999, 77.

(14) Ananthakrishnan, V.; Gill, W. N.; Allen, J. B. Laminar Dispersion in Capillaries: Part I. Mathematical Analysis. AIChE Journal 1965, 11, 1063-1072.

(15) Levenspiel, O. Chemical Reaction Engineering, 3rd ed.; John Wiley \& Sons, 1999.

(16) Nyande W., B.; Thomas, M. K.; Lakerveld, R. CFD Analysis of a Kenics Static Mixer with a Low Pressure Drop under Laminar Flow Conditions. Industrial 8$\}$ Engineering Chemical Research 2021, 60, 5264-5277.

(17) Rojhan, P.; Hessel, V.; Nigam, K. D. P.; Schael, F. Applicability of the axial dispersion model to coiled flow inverters containing single liquid phase and segmented liquid-liquid flows. Chem. Eng. Sci. 2018, 182, 77-92.

(18) Florit, F.; Rota, R.; Jensen, K. F. Dispersion in coiled tubular reactors: A CFD and experimental analysis on the effect of pitch. Chemical Engineering Science 2021, 233, 116393.

(19) Klutz, S.; Kurt, S., Kutup; Lobedann, M.; Kockmann, N. Narrow residence time distribution in tubular reactor concept for Reynolds number range of 10-100. Chemical Engineering Research and Design. 2015, 95, 22-23.

(20) Montante, G.; Coroneo, M.; Paglianti, A. Blending of miscible liquids with different 
densities and viscosities in static mixers. Chemical Engineering Science 2016, 141, 250-260.

(21) Thakur, R.; Vial, C.; Nigam, K.; Nauman, E.; Djelveh, G. Static mixers in the process industries - a review. Chemical Engineering Research and Design 2003, 81, 787-826.

(22) Albertazzi, J.; Florit, F.; Busini, V.; Rota, R. Mixing Efficiency and Residence Time Distributions of a Side- Injection Tubular Reactor Equipped with Static Mixers. Industrial \& Engineering Chemical Research 2021,

(23) Regner, M.; Ostergren, K.; Trägårdh, C. Influence of Viscosity Ratio on the Mixing Process in a Static Mixer: Numerical Study. Industrial and Engineering Chemistry Research 2008, 47, 3030-3036.

(24) Mukhopadhyay, G. L. A.; Yi Dai, C.-Y. C. Various approaches to compute fluid residence time in mixing systems. Proceedings of the ASME 2010 3rd Joint US-European Fluids Engineering Summer Meeting and 8th International Conference on Nanochannels, Microchannels, and Minichannels. 2010.

(25) ANSYS Inc., ANSYS Fluent Theory Guide - release 19.1; 2018.

(26) Hirschberg, S.; Koubek, R.; Moser, F.; Schöck, F. An improvement of the Sulzer $\mathrm{SMX}^{T M}$ static mixer significantly reducing the pressure drop. Chemical Engineering Research and Design 2009, 87, 524-532.

(27) Paul L., E.; Atiemo-Obeng, V. A.; Kresta, S. M. Handbook of Industrial Mixing; 2002.

(28) Leach, S. Stratification and Mixing of fluids of different densities. I.Chem.E. Symposyum Series 1968, 25, 17-25. 